\title{
Raquitismo: Ressurgimento do Passado
}

\author{
Rickets: Emerging From the Past
}

\begin{abstract}
Margarida VALÉRIO ${ }^{1}$, Sara PIMENTEL MARCOS ${ }^{1}$, Conceição SANTOS ${ }^{1}$, Maria João LEIRIA ${ }^{1}$
Acta Med Port 2015 Mar-Apr;28(2):263-266

RESUMO

Na última década o raquitismo tem ressurgido nos países desenvolvidos, devido a alterações no estilo de vida e hábitos alimentares. Descrevemos o caso de uma criança de 28 meses, raça negra, antecedentes de aleitamento materno exclusivo até aos nove meses sem suplementação vitamínica e ausência de ingestão de produtos lácteos por suspeita de intolerância às proteínas de leite de vaca, internado por má progressão estaturo-ponderal. Apresentava varismo acentuado, rosário costal e punhos de boneca. Analiticamente destacava-se elevação da paratormona intacta e fosfatase alcalina, com diminuição do calcidiol. O estudo radiológico evidenciava desmineralização óssea generalizada, com alargamento das extremidades e metáfises do rádio e cúbito distais em forma de taça. Admitido o diagnóstico de raquitismo carencial, foi instituída terapêutica com colecalciferol e carbonato de cálcio, com progressiva melhoria clínica, laboratorial e radiológica. Neste caso destacam-se vários fatores que, conjugados, contribuíram para um raquitismo carencial grave, alertando para o ressurgir desta patologia.
\end{abstract}

Palavras-chave: Criança; Deficiência de Vitamina D; Raquitismo; Transtornos da Nutrição Infantil.

\section{ABSTRACT}

In the past decade rickets has re-emerged in developed countries due to changes in lifestyles and dietary habits. We describe a case of a 28-month-old black infant with failure to thrive. He was exclusively breastfed until nine months of age, without vitamin supplementation, and never ingested milk products due to alleged cow's milk intolerance. His examination revealed bowlegs, rachitic rosary and wide wrists. Alkaline phosphatase and intact parathyroid hormone levels were elevated, and calcidiol was decreased. Radiographic images showed bone demineralization, fraying and cupping of the distal radius and ulna. Nutritional rickets was considered and treatment with colecalciferol and calcium carbonate was initiated, with clinical, laboratory and radiologic improvement. In this case, a group of factors contributed to severe nutritional rickets, alerting to the re-emergence of this disease.

Keywords: Child; Child Nutrition Disorders; Rickets; Vitamin D Deficiency.

\section{INTRODUÇÃO}

$O$ raquitismo é uma patologia resultante de um defeito de mineralização do tecido osteoide do osso em crescimento. ${ }^{1}$ De acordo com a etiologia pode classificar-se em três grupos: carencial (défice de aporte), primário (defeitos congénitos do metabolismo da vitamina $D$ ), ou secundário (sendo o raquitismo hipofosfatémico familiar o mais frequente). ${ }^{1}$

Com elevada prevalência até há três décadas, o raquitismo carencial permanece um problema de saúde pública nos países desenvolvidos, onde tem ressurgido na última década devido a alterações no estilo de vida e hábitos alimentares. ${ }^{2-4}$ Países como Canadá e Estados Unidos da América (EUA) têm reportado na última década inúmeros casos clínicos e elaborado revisões casuísticas que revelam um aumento importante dos casos de raquitismo carencial. ${ }^{3-5}$ Dos fatores de risco identificados destacam-se, pela sua prevalência, exposição solar insuficiente, deficiência materna de vitamina $D$, raça negra e aleitamento materno exclusivo e por períodos prolongados (além dos seis meses de idade). ${ }^{2-5} \mathrm{~A}$ prevenção destas situações, com a promoção de uma diversificação alimentar correta e suplementação vitamínica adequada, bem como a identificação precoce de crianças em risco, pode evitar defeitos e repercussões graves na mineralização óssea. ${ }^{1}$
Descreve-se um caso clínico de uma criança com raquitismo carencial grave, seguida de uma breve revisão da literatura a respeito do tema.

\section{CASO CLÍNICO}

Criança do sexo masculino, raça negra, internada aos 28 meses para estudo de má progressão estatural, por dúvidas na adesão à investigação em consulta. Antecedentes pessoais de gestação de termo, aleitamento materno exclusivo até aos nove meses, sem suplementação vitamínica. Diversificação alimentar com ausência de ingestão de produtos lácteos por suspeita de intolerância às proteínas de leite de vaca (PLV). Constatou-se atraso do desenvolvimento estatural, com desaceleração da velocidade de crescimento a partir dos 15 meses, com cruzamento do percentil 75 para o 10; verificou-se concomitante descida do percentil 50 para o 25 no peso (de acordo com as curvas de crescimento da OMS). Antecedentes familiares irrelevantes. Ao exame objetivo apresentava varismo acentuado, rosário costal, punhos de boneca e eczema atópico. No estudo analítico (Tabela 1) destacava-se normocalcemia, hipofosfatemia, elevação da paratormona intacta $(\mathrm{PTHi})$ e da fosfatase alcalina (FA), diminuição de calcidiol (25[OH]-D), hipocalciúria e hipofosfatúria. Rastreio de doença celíaca

1. Serviço de Pediatria. Hospital de São Francisco Xavier. Centro Hospitalar de Lisboa Ocidental. Lisboa. Portugal.

Recebido: 20 de Junho de 2014 - Aceite: 16 de Fevereiro de 2015 | Copyright @ Ordem dos Médicos 2015 
Tabela 1 - Evolução laboratorial

\begin{tabular}{|c|c|c|c|c|c|c|}
\hline & Admissão & $\begin{array}{l}6 \text { Semanas de } \\
\text { tratamento }\end{array}$ & $\begin{array}{l}6 \text { Meses de } \\
\text { tratamento }\end{array}$ & $\begin{array}{l}8 \text { Meses de } \\
\text { tratamento }\end{array}$ & $\begin{array}{l}14 \text { Meses de } \\
\text { tratamento }\end{array}$ & Valores Ref* \\
\hline Cálcio (S) & 9,1 & 9,3 & 9,8 & 9,9 & 10 & $8,8-10,8 \mathrm{mg} / \mathrm{dL}$ \\
\hline Fósforo (S) & 3,0 & 5,3 & 5,9 & 6,3 & 6,6 & $4,5-9,0 \mathrm{mg} / \mathrm{dL}$ \\
\hline Vit. B12 & $<117$ & 184 & & & & 176-687 pmol/L \\
\hline 25(OH)-D & 12 & 15 & 27 & 23 & 81 & $75-250 \mathrm{nmol} / \mathrm{L}$ \\
\hline PTHi & 72,3 & 102,1 & 19,2 & 17,3 & 12,1 & $15-68,3 \mathrm{pg} / \mathrm{mL}$ \\
\hline FA & 735 & 550 & 241 & 240 & 223 & $145-320 \mathrm{U} / \mathrm{L}$ \\
\hline Cálcio (U) & 8 & 9,2 & & & 68 & $42-353 \mathrm{mg} / 24 \mathrm{~h}$ \\
\hline Fósforo (U) & 3,3 & 4,2 & & & 8,5 & $4-13 \mathrm{mg} / 24 \mathrm{~h}$ \\
\hline
\end{tabular}

${ }^{\star}$ Fonte: Videira Amaral G. Tratado de Clínica Pediátrica; $2^{a}$ edição. 2013. p. 2086-2091

negativa. Radiologicamente evidenciava desmineralização óssea generalizada, com alargamento das extremidades dos membros e metáfises em forma de taça (Fig. 1). Dosearam-se IgE específicas para PLV na classe 2. Efetuou prova de tolerância oral ao leite, sem reação de hipersensibilidade. Admitido o diagnóstico de raquitismo carencial grave foi instituída terapêutica com colecalciferol $6003 \mathrm{UI} /$ dia per os (PO) associado a carbonato de cálcio $500 \mathrm{mg} / \mathrm{dia}$

\section{PO durante seis semanas.}

Manteve seguimento em consulta de Pediatria com monitorização clínica, laboratorial (Tabela 1) e radiológica. Às seis semanas de tratamento, por fraca resposta à terapêutica e suspeita de má adesão, aumentou-se a dose de colecalciferol para 6670 Ul/dia. Verificou-se regressão progressiva e completa das deformidades ósseas, bem como normalização dos parâmetros analíticos, aos catorze

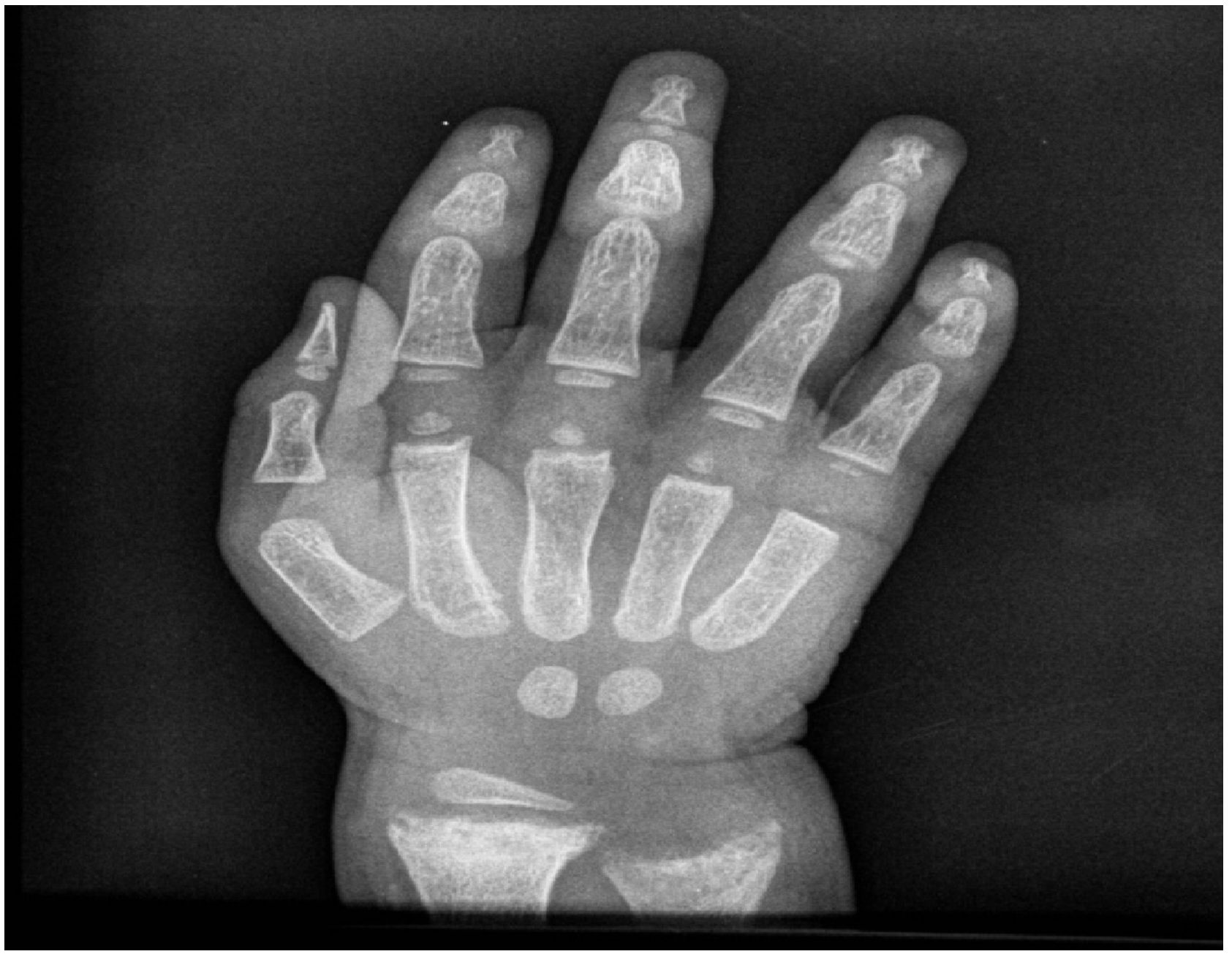

Figura 1 - Radiografia de punho na admissão 
meses de terapêutica. Radiologicamente, aos seis meses já havia evidência de melhoria (Fig. 2), com resolução completa aos treze meses.

Teve alta da consulta ao fim de dois anos, cumprindo terapêutica de manutenção com colecalciferol (667 UI/dia) desde os doze meses de seguimento.

\section{DISCUSSÃO}

O raquitismo carencial resulta de exposição solar insuficiente ou ingestão inadequada de vitamina $D$, cálcio ou fósforo. ${ }^{1,2,6} A$ apresentação clínica é variável e os sinais incluem alargamento das epífises dos ossos longos, deformações dos membros inferiores, proeminência das articulações condrocostais, entre outras. ${ }^{1,6,7}$ Quanto à semiologia radiológica as alterações incluem perda de nitidez da linha metafisária, alargamento da placa de crescimento e desmineralização óssea. ${ }^{1,6,7}$ Laboratorialmente destaca-se hipo ou normocalcemia, hipofosfatemia, aumento da FA e PTHi, e diminuição de $25(\mathrm{OH})-\mathrm{D} .^{1,6,7} \mathrm{~A}$ terapêutica consiste na reposição de vitamina $D$ e cálcio, com a posologia inicial adequada à idade, com duração de oito a doze semanas, seguida de terapêutica de manutenção. ${ }^{6-9}$ Deve ser mantido um seguimento regular durante 12 meses, com monitorização clínica, analítica e radiológica. ${ }^{6,7}$ As deformidades ósseas, bem como as alterações analíticas e radiológicas, regridem por completo após terapêutica médica dirigida, geralmente ao fim de seis meses. ${ }^{6,7,10}$ Neste caso, com a suspeita de má adesão à terapêutica, nomeadamente com faltas consecutivas ás consultas e demora na normalização dos valores laboratoriais apesar da terapêutica dirigida instituída, poderíamos ter optado pela stosstherapy, ${ }^{9}$ dose única intramuscular ou PO de 600000 IU de vitamina D ou dose semanal de 60000 IU PO durante dez semanas, seguida de terapêutica de manutenção (400 IU/dia). ${ }^{9}$

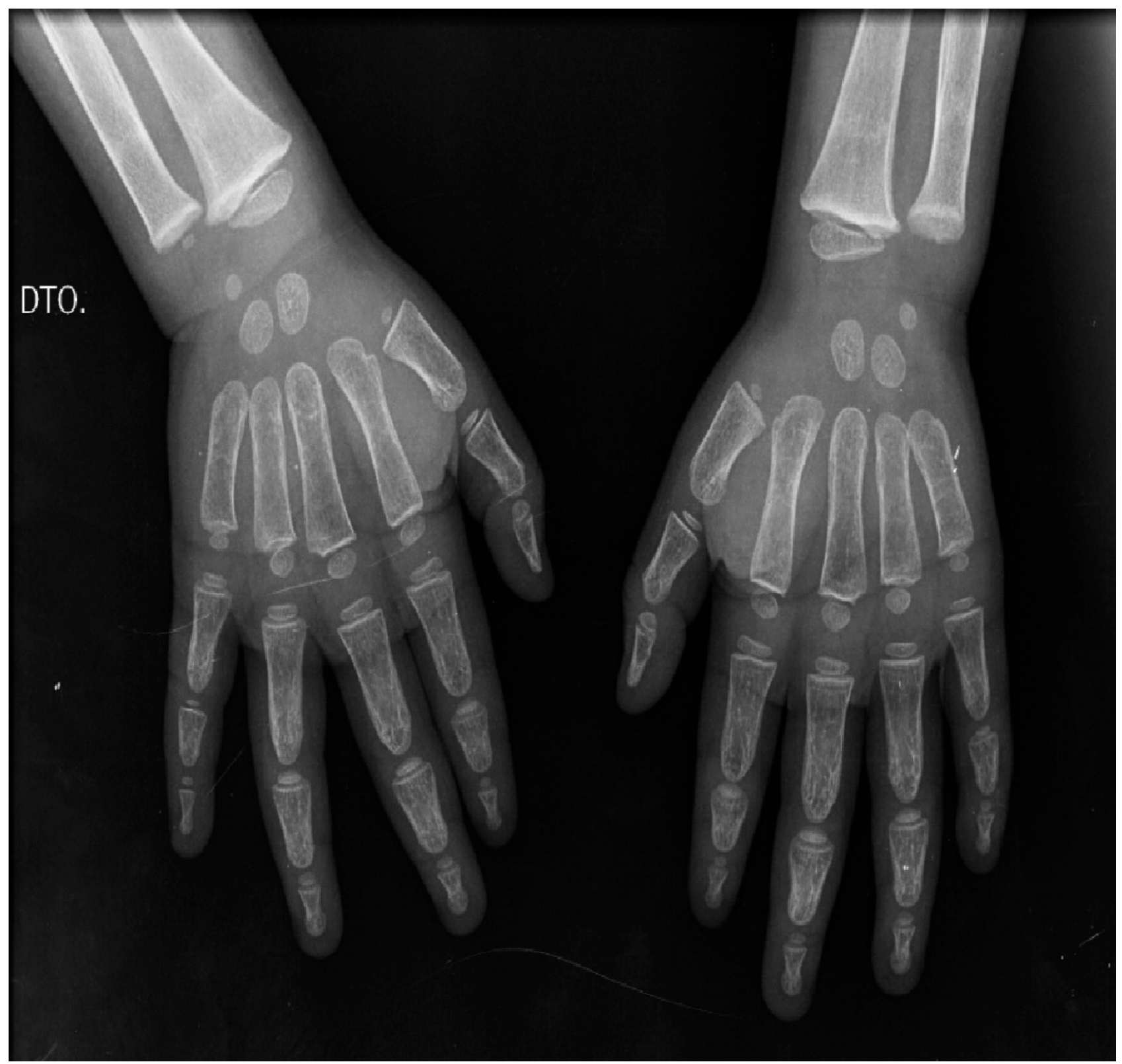

Figura 2 - Radiografia de punho ao fim de 6 meses de tratamento 
Neste caso não optámos por esta terapêutica por apresentar melhoria progressiva das alterações analíticas e radio-

Apesar da preocupação relativamente à persistência e potencial aumento da incidência de raquitismo carencial nos países desenvolvidos, a sua incidência atual é desconhecida. ${ }^{2,3,5}$ Nos EUA os casos publicados de raquitismo carencial aumentaram de 65 entre 1975 e 1985 para 166 casos entre 1986 e $2003,{ }^{3}$ facto que alertou a Academia Americana de Pediatria em 2003 a recomendar a suplementação com vitamina D (200 IU/dia); em 2008 a recomendação mudou para $400 \mathrm{UI} / \mathrm{dia}$, sendo abrangidos por esta recomendação todos os lactentes em aleitamento materno exclusivo, misto e com ingestão diária de fórmula para lactentes inferior a um litro. $5,7,12$ Esta dose foi igualmente aumentada em países como Portugal, Canadá, Inglaterra e Austrália. ${ }^{4,7,13}$

Um dos principais fatores responsáveis pelo reaparecimento do raquitismo carencial é a exposição solar insuficiente, associada às recomendações do uso em idade pediátrica de protetores solares com elevado índice para os raios UV-B, essenciais para a síntese cutânea de vitamina $D$, e ao uso excessivo de roupa bloqueando a penetração da luz solar. ${ }^{11,12}$ Crianças de raça negra são mais suscetíveis pois a sua melanina bloqueia a entrada dos raios UV-B na pele. ${ }^{7,8,12}$ Outros fatores de risco importantes são a defi-

\section{REFERÊNCIAS}

1. Martins de Pina RM. Raquitismo. In: Palminha JM, Carrilho EM, editores. Orientação diagnóstica em Pediatria Volume I. Lisboa: Lidel; 2003. p. 569-76.

2. Prentice A. Nutritional rickets around the world. J Steroid Biocherm Mol Biol. 2013;136:201-6

3. Weisberg P, Scanlom KS, Li R, Cogswell ME. Nutritional rickets among children in the United States: review of cases reported between 1986 and 2003. Am J Clin Nutri. 2004;80:1697S-705S.

4. Ward LM, Gaboury I, Ladhani M, Zlotkin S. Vitamin D-deficiency rickets among children in Canada. CMAJ. 2007;177:161-6.

5. Thatcher TD, Fischer PR, Tebben PJ, Singh RJ, Cha SS, Maxson JA, et al. Increasing incidence of nutritional rickets: a population-based study in Olmsted County, Minnesota. Mayo Clin Proc. 2013;88:176-83.

6. Nield LS. Rickets: not a disease of the past. Am Fam Phys. 2006;74:61926.

7. Misra M, Pacaud D, Petryk A, Collett-Solberg PF, Kappy M. Vitamin D deficiency in children and its management: review of current knowledge and recommendations. Pediatrics. 2008;122:398-415.

8. Lee Y, So TY, Thackray J. Review on vitamin D deficiency treatment in ciência materna de vitamina $\mathrm{D}^{7,8,12}$ e o aleitamento materno exclusivo por períodos prolongados ${ }^{7,8,12,14}$ (o leite materno é pobre em vitamina $D,{ }^{15}$ sendo fundamental a suplementação vitamínica a partir do primeiro mês de vida). ${ }^{7,12}$

Para este caso clínico em concreto contribuíram várias variáveis que culminaram no raquitismo carencial grave: aleitamento materno exclusivo sem suplementação vitamínica, raça negra e erros alimentares, com ausência de ingestão de leite e derivados. Pretendemos alertar para a necessidade de equacionar novamente esta patologia, destacando a importância da prevenção destas situações, com a promoção de uma diversificação alimentar correta e suplementação vitamínica adequada.

\section{OBSERVAÇÕES}

Apresentado como Comunicação Oral no Congresso Nacional de Pediatria em Outubro de 2011.

\section{CONFLITOS DE INTERESSE}

Os autores declaram não ter nenhum conflito de interesse relativamente ao presente artigo.

\section{FONTES DE FINANCIAMENTO}

Não existiram fontes externas de financiamento para a realização deste artigo.

pediatric patients. J Pediatric Pharmacol Ther. 2013;18:277-91.

9. Mondal K, Seth A, Marwaha RK, Dhanwal D, Aneja S, Singh R, et al Randomized controlled trial on safety and efficacy of single intramuscular versus staggered oral dose of $600000 \mathrm{UI}$ Vitamin D in treatment of nutritional rickets. J Trop Pediatr. 2014;59:105-13.

10. Chatterjee D, Gupta V, Sharma V, Sinha B, Samanta S. A reliable and cost effective approach for radiographic monitoring in nutritional rickets. J Radiol. 2014;87:20130648.

11. Lowdon J. Rickets: concerns over the worldwide increase. J Fam Health Care. 2011;21:25-9.

12. Wagner C, Greer FR. Prevention of rickets and vitamin D deficiency in infants, children and adolescents. Pediatrics. 2008;122:1142-52.

13. Comissão de Nutrição da Sociedade Portuguesa de Pediatria Alimentação e nutrição do lactente. Acta Pediatr Port. 2012:43:S17-40.

14. Specker BL, Valanis B, Hetzberg V, Edwards N, Tsang RC. Sunshine exposure and serum 25.hidroxyvitamin $D$ concentrations in exclusively breast-fed infants. J Pediatr. 1985;107:372-6.

15. Leerbeck $E$, Sondergaard $H$. The total content of vitamin $D$ in human milk and cow's milk. Br J Nutr. 1980;44:7-12. 


\section{Margarida VALÉRIO, Sara PIMENTEL MARCOS, Conceição SANTOS, Maria João LEIRIA \\ Raquitismo: Ressurgimento do Passado}

Acta Med Port 2015:28:263-266

Publicado pela Acta Médica Portuguesa, a Revista Científica da Ordem dos Médicos

Av. Almirante Gago Coutinho, 151

1749-084 Lisboa, Portugal.

Tel: +351218428 215

E-mail: submissao@actamedicaportuguesa.com

www.actamedicaportuguesa.com

ISSN:0870-399X | e-ISSN: 1646-0758

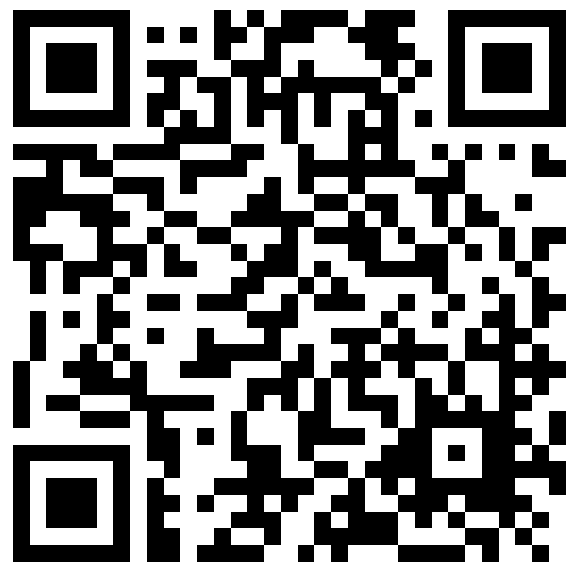

